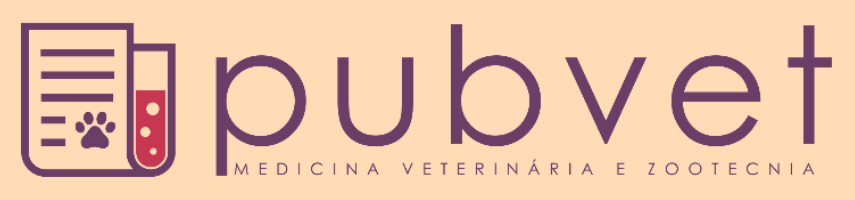

https://doi.org/10.31533/pubvet.v14n7a603.1-7

\title{
Aspectos relacionados ao potencial forrageiro do sorgo: Revisão
}

\author{
Patrick Bezerra Fernandes $^{1 *} \bullet \mathbb{D}$, Gustavo de Faria Theodoro ${ }^{\circ} \oplus$ (D), Antonio Leandro Chaves

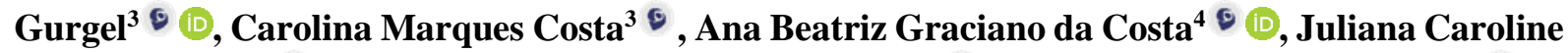 \\ Santos Santana $^{4} \bullet \mathbb{D}$, Manoel Gustavo Paranhos da Silva ${ }^{4} \bullet$ (D), Leandro Nogueira Bomfim ${ }^{\circ}$
}

${ }^{I}$ Doutor em Ciência Animal - Universidade Federal de Mato Grosso do Sul

${ }^{2}$ Professor do Programa de Pós-Graduação em Ciência Animal - Universidade Federal de Mato Grosso do Sul

${ }^{3}$ Aluno do Curso de Doutorado em Ciência Animal - Universidade Federal de Mato Grosso do Sul

${ }^{4}$ Aluno do Curso de Mestrado em Ciência Animal - Universidade Federal de Mato Grosso do Sul

${ }^{5}$ Aluno do Curso de Zootecnia- Universidade Federal de Mato Grosso do Sul

*Autor para correspondência, E-mail: zoo.patrick@hotmail.com

Resumo. Para a produção de pequenos e grandes ruminantes em regiões de clima tropical, torna-se necessário buscar fontes de alimentos que supram a necessidade dos animais em pastejo ou plantas forrageiras suplementares, agindo como uma forma estratégica de potencializar a produção animal nessas regiões. O sorgo apresenta características favoráveis para regiões de clima tropical, pois exibe rápido estabelecimento, resistência ao déficit hídrico e, comparado às demais culturas, apresenta baixa exigência em fertilidade do solo, sendo uma excelente alternativa para regiões de clima tropical, em que há baixos níveis de fertilidade natural, além da estacionalidade de produção de forragem. Apesar de ser uma cultura de ampla aptidão, o potencial do sorgo é pouco explorado, pois é possível encontrar linhagens com potencial para a produção de grão, materiais forrageiros para silagem e pastejo. Diante disso, foi elaborada uma revisão com os aspectos relacionados ao potencial forrageiro do sorgo. O sorgo apresenta bons índices para a produção de animais a pasto, assim como em situações que almejam a utilização como fonte de forragem suplementar, no entanto, ainda há necessidade de maiores incentivos para a utilização desse material forrageiro na alimentação animal.

Palavras chave: adubação, morfogênese, ruminante

\section{Aspects related to the forage potential of sorghum: Review}

Abstract. For the production of small and large ruminants in regions of tropical climate, it is necessary to seek sources of food that supply the need for animals in paste or supplementary forage plants, as a strategic way to enhance animal production in these situations. It has favorable characteristics for tropical climatic regions, rapid rapid tests, resistance to water deficits and, compared to other crops, it presents low soil fertility rates, being an excellent alternative for tropical climatic regions, where there are low natural fertility values, besides the seasonality of forage production. Despite being a large capacity crop, the potential for sorghum is little explored, it is possible to find lines with potential for grain production, forage materials for silage and paste. Therefore, a review was prepared with aspects related to the forage potential of sorghum. Sorghum has good rates for the production of animals on pasture, as well as in situations that are also used as a source of supplementary fodder, however, there is still a need for greater incentives for the use of this forage material in animal feed.

Keywords: fertilization, morphogenesis, ruminant 


\title{
Aspectos relacionados al potencial forrajero del sorgo: Revisión
}

\begin{abstract}
Resumen. Para la producción de pequeños y grandes rumiantes en regiones de clima tropical, es necesario buscar fuentes de alimentos que satisfagan la necesidad de los animales en pastoreo o plantas de forraje suplementarias, como una forma estratégica de mejorar la producción animal en estos territorios. El sorgo tiene características favorables para las regiones climáticas tropicales, pruebas rápidas, resistencia al déficit hídrico y, en comparación con otros cultivos, presenta tasas bajas de fertilidad del suelo, siendo una excelente alternativa para las regiones climáticas tropicales, donde hay bajos valores de fertilidad natural, además de la estacionalidad de la producción de forraje. A pesar de ser un cultivo de gran capacidad, el potencial para el sorgo es poco explorado, es posible encontrar líneas con potencial para la producción de granos, materiales forrajeros para ensilaje y pastoreo. Por lo tanto, se preparó una revisión con aspectos relacionados con el potencial de forraje del sorgo. El sorgo tiene buenas tasas para la producción de animales en pastoreo, así como en situaciones que también se usan como fuente de forraje suplementario, sin embargo, todavía existe la necesidad de mayores incentivos para el uso de este material forrajero en la alimentación animal.
\end{abstract}

Palabras clave: fertilización, morfogénesis, rumiante

\section{Introdução}

Para a produção de pequenos e grandes ruminantes em regiões de clima tropical, torna-se necessário buscar fontes de alimentos que supram a necessidade dos animais em pastejo ou plantas forrageiras suplementares, como forma estratégica de potencializar a produção animal nessas regiões. Por outro lado, em condições de Brasil central, sendo uma região promissora para a produção animal, a vegetação nativa não é adaptada para o pastejo ou corte. Sendo assim, é necessária a escolha de culturas forrageiras adaptadas para as regiões de cerrado. Atualmente, as pastagens cultivadas utilizadas que apresentam potencial forrageiro são as gramíneas dos gêneros Brachiaria e Panicum. Esses dois gêneros não são capazes de suprir a demanda de forragem ao longo do ano, pois sofrem com a escassez de chuva (Barbosa et al., 2007, 2011; Euclides et al., 2008; Fernandes et al., 2020). Além disso, as cultivares de Panicum são extremamente exigentes em fertilidade do solo, por isso torna-se necessário a associação e interação com novos matérias forrageiros para equilibrar a produção de forragem.

O sorgo como cultura forrageira vêm preenchendo a necessidade de forragem verde durante o verão, pois apresenta melhor eficiência do uso da água e adaptações resistentes à seca, além de menor necessidade de fertilizantes (Rosa et al., 2004; Bhat, 2019). É comumente utilizado no estágio vegetativo para suprir as necessidades de produção de forragem no verão pelo pastoreio o sistema de corte e transporte.

Atualmente, existem três tipos principais de sorgo cultiváveis: o sorgo forrageiro, produz mais matéria seca que o sorgo grão, possui caules grossos e é muito utilizado para ensilagem (Rezende et al., 2020; Ribeiro et al., 2017), o sorgo granífero, que apresenta potencial intermediário de produção e pode ser usado para pastagem, feno ou silagem (Ribeiro et al., 2015), e o sorgo hibrido com capim-sudão, que apresenta caule fino, excelente capacidade de perfilhamento e regeneração após o pastejo (Bath, 2019; Simili et al., 2011).

No entanto, o uso do sorgo como potencial forrageiro é afetado pela falta de informações sobre seu uso na alimentação animal. Diante disso, foi elaborada uma breve revisão com os aspectos relacionados ao potencial forrageiro do sorgo.

\section{Escolha do híbrido e o efeito da adubação}

A escolha de materiais genéticos que possam vir a aumentar a produtividade do sistema requer pesquisas para identificação de híbridos promissores que apresentem características morfológicas e estruturais que sejam de fácil adaptação as condições ambientais da região de cultivo (Macêdo et al., 2018). Além disso, é necessário que a escolha do hibrido se correlacione positivamente com a produção de massa de forragem e características de perfilhamento. 
Nitrogênio $(\mathrm{N})$, fósforo $(\mathrm{P})$ e potássio $(\mathrm{K})$ são os três nutrientes essenciais que afetam o rendimento e qualidade do sorgo. $\mathrm{O} \mathrm{N}$ é muito importante por promover altas taxas de crescimento, produtividade e qualidade, devido ao seu papel na divisão celular (Wang et al., 2017). Isso pode ser verificado quando há mensuração em nível de perfilho individual, pois mesmo sabendo que as variáveis responsáveis pelo acúmulo de biomassa são geneticamente determinadas (Lemaire et al., 2009) foi observado em híbridos de sorgo, que a medida que duplicava a dose de $\mathrm{N}$, provocava variações nas características no fluxo de tecidos (Silva et al., 2012) (Figura 1).

Mazzanti et al. (1994) relatam que as variáveis relacionadas à morfogênese apresentam uma forte relação com a adubação, assim, à medida que o efeito da adubação aumenta o processo de divisão celular para a renovação de órgãos (Figura 1A), provoca a redução no intervalo entre o aparecimento entre duas folhas (filocrono, Figura 1B), e com isso impacta em aceleração no fluxo de tecidos, pois reduz a duração de vida de folha (DVF; Figura 1D).
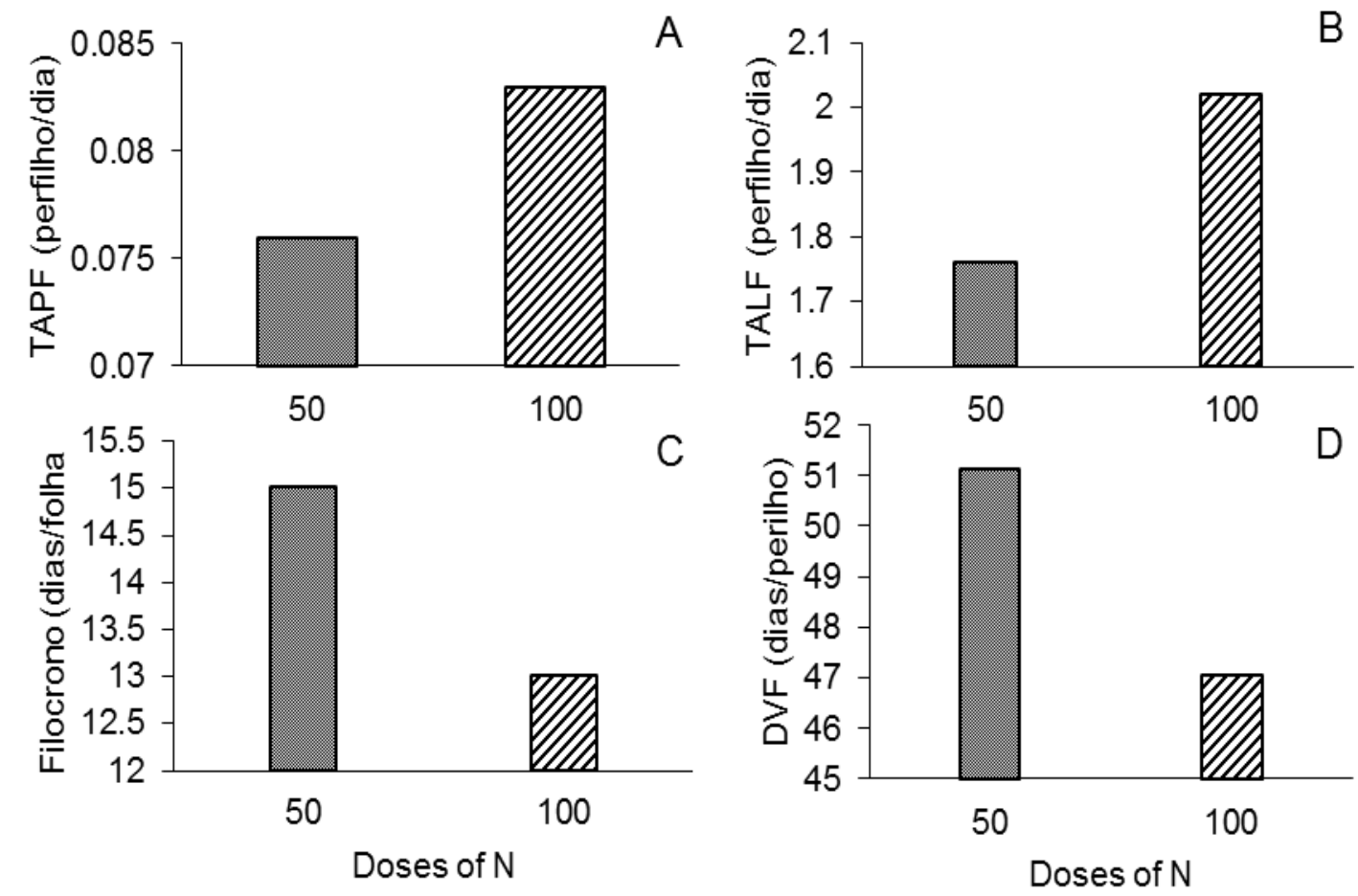

Figura 1. A: Taxa de aparecimento de folha (TAPF, folhas perfilho/dia); B: Taxa de alongamento de folha (TAlF, cm de lâmina foliar/perfilho/dia), C: Filocrono (Filoc, dias folha/perfilho), D: Duração de vida das folhas (DVF, dias), em função das duas doses de adubação nitrogenada, adaptado de Silva et al. (2012).

Para Gastal \& Lemaire (2015) e Menezes et al. (2019) essas respostas já eram esperadas, além disso, o $\mathrm{N}$ potencializa a absorção de carbono atmosférico, devido o ganho em área foliar (Figura 1B). Por outro lado, os autores salientam que o grande entrave desse acréscimo na produção está na forma como será realizado o aproveitamento de forma eficiente do produto forrageiro gerado, sendo necessário que ocorra a desfolhação de forma eficiente antes de ocorrer a senescência da forragem produzida (Fernandes et al., 2020; Oliveira et al., 2020). Por outro lado, quando qualquer planta com potencial forrageiro é submetida a alguma situação de pastejo pelo animal, isso causa efeitos sobre o fluxo de tecidos (Difante et al., 2011; Montagner et al., 2011). Diante disso torna-se pertinente verificar a interação entre o manejo imposto aliado ao nível de adubação, além dos demais nutrientes essenciais para o desenvolvimento da planta.

$\mathrm{O}$ fosforo $(\mathrm{P})$ influencia o crescimento e rendimento das culturas, aumentando a taxa de crescimento de todos as partes da cultura após a emergência, e também está intimamente relacionado a síntese do amido, muito importante para produção de sorgo granífero (Wang et al., 2017). O potássio (K) está entre os nutrientes essenciais para o bom desenvolvimento das plantas de sorgo, além de poder impactar de forma positiva no acréscimo de biomassa. Além disso, o K melhora a captação de água pelas raízes, 
promove fotossíntese e aumenta a capacidade do sorgo em suportar o estresse (Wang et al., 2017). Segundo Galvão et al. (2015), quando se trabalha com doses de 100 a $150 \mathrm{~kg} / \mathrm{ha}$ de K, é possível obter em média 10 toneladas de massa de forragem.

Híbridos de Sorghum bicolor cv. Sudanense manejados em lotação intermitente, estabelecendo-se como critério altura de entrada de $1,0 \mathrm{~m}$ e a saída de resíduo de $0,50 \mathrm{~m}$, para essa situação foi observado que a adubação de manutenção necessária apenas $100 \mathrm{~kg} / \mathrm{ha}$ de $\mathrm{P}_{2} \mathrm{O}_{5}, 80 \mathrm{~kg} / \mathrm{ha}$ de $\mathrm{K}_{2} \mathrm{O} 100 \mathrm{~kg} / \mathrm{ha}$ de N, seria suficiente para manter o valor nutritivo, e valores superiores a esses não acarretaria em acréscimos na porcentagem de matéria seca dos componentes massa de lâmina foliar e massa de pseudocolmo ( $\underline{\text { Silva }}$ et al., 2012; Simili et al., 2008).

\section{Sorgo para pastejo}

O sorgo pode ser utilizado em situações em que se pretende fazer a colheita da forragem produzida por meio da desfolhação realizada pelo animal, no entanto é primordial a escolha híbridos que apresentem uma rápida rebrota e um aceleramento na taxa de aparecimento de perfilhos, por ser uma cultura anual a necessidade de maior potencialização de utilização do material forrageiro produzido. Uma das formas de melhorar o aproveitamento do sorgo seria em sistema de lotação rotacionada, em que por meio dessa estratégia é possível controlar o fluxo de tecidos, especialmente a taxa de alongamento de colmo (Barbosa et al., 2011; Santos et al., 2011).

O ideal é que se trabalhe com intervalo de pastejo inferior a 40 dias, pois maiores espaços entre os ciclos de utilização, apesar do ganho em biomassa total, reduz o valor nutritivo do pasto, e proporção de massa de folha na massa de forragem, impactando negativamente na colheita de forragem (Fonseca et al., 2012), pois a medida que ocorre aumento na distribuição horizontal de massa de colmo, aumenta o tempo de ruminação, acarretando em perdas na produção animal, uma vez que o colmo é a fração da massa de forragem que apresentar menor valor nutritivo (Benvenutti et al., 2008, 2009).

Em lotação continua o sorgo forrageiro apresenta bons índices agronômicos e zootécnicos (Pacheco et al., 2014; Rodrigues et al., 2017) (Tabela 1), em que apesar da variação na taxa de lotação e na massa de forragem, é observado semelhança no ganho de peso entre as cultivares de sorgo.

Tabela 1. Massa de forragem (MF, T/ha), proteína bruta (\%, PB), fibra em detergente neutro (\%, FDN), ganho médio diário (GMD kg/dia) e taxa de lotação (TL, $\left.{ }^{1} \mathrm{UA} / \mathrm{ha}\right)$.

\begin{tabular}{|c|c|c|c|c|c|c|c|}
\hline Autores & Gramínea & $\mathrm{MF}$ & Dias & PB & FDN & GMD & TL \\
\hline${ }^{2}$ Pacheco et al. (2014) & Sorghum bicolor $\mathrm{cv}$. sudanense & 1,6 & 63 & 13,0 & 50,5 & 0,960 & 4.2 \\
\hline${ }^{2}$ Rodrigues, et al. (2017) & Sorghum bicolor L. Moench & 5,5 & 84 & 16,0 & 70,0 & 0,918 & 2,6 \\
\hline${ }^{3}$ Euclides et al. (2007) & Brachiaria decumbens & 3,8 & 60 & 8,5 & 70,5 & - & 3,0 \\
\hline \multirow{3}{*}{$\underline{ }{ }^{4}$ Euclides et al. (2008) e Euclides et al. (2009) } & Brachiaria brizantha cv. Marandu & 3,1 & 180 & 8,2 & 70,1 & 0,740 & 2,7 \\
\hline & Brachiaria brizantha cv. Piatã & 3,2 & 180 & 8,2 & 73,0 & 0,770 & 2,9 \\
\hline & Brachiaria brizantha cv. Xaraés & 3,3 & 180 & 8,2 & 72,9 & 0,675 & 3,8 \\
\hline
\end{tabular}



As gramíneas do gênero Brachiaria são mais utilizadas nas fases de cria e recria, além de serem pastos perenes, porém não consegue potencializa o ganho de peso do animal (Tabela 1), uma estratégia a ser considerada, seria realizar a preparação dos animais em pastos perenes, e a terminação a pasto utilizando o sorgo forrageiro. Uma vez que, entre as variáveis apresentadas (Tabela 1), e possível inferir que em curto espaço de tempo o sorgo pode promover a mesma disponibilidade de forragem e até mesmo, maior produtividade por área quando se faz o produto da taxa de lotação e ganho médio diário.

\section{Sorgo para silagem}

Devido à redução na produção de forragem nas épocas secas do ano associada as baixas temperaturas, umidade e luz, o planejamento e implementação de práticas voltadas conservação de forragem, como a silagem, é uma opção que tem sido amplamente utilizado na alimentação de ruminantes (Ribeiro et al., 2015, 2017).

Entre as culturas que podem ser utilizadas para a conservação de forragem, o sorgo (Sorghum bicolor) apresenta potencial, devido suas características agronômicas (Ali et al., 2009), além dos bons 
índices zootécnicos (Tabela 2), podendo ser potencialmente mais digestível que outras plantas forrageiras (Pinto et al., 2010).

Tabela 2. Proteína bruta (\%, PB), fibra em detergente neutro (\%, FDN), ganho médio diário (GMD kg/dia).

\begin{tabular}{lcccc}
\hline Autores & Gramínea & PB & FDN & GMD \\
\hline Neumann et al. (2002) & Sorghum bicolor, L. Moench & 11,2 & 58.8 & 0,943 \\
Chieza et al. (2008) & Sorghum bicolor, L. Moench & 6,6 & 60,5 & 1,42 \\
\hline$\underline{\text { Pinto et al. (2010) }}$ & Silagem de sorgo & 12,51 & 40,4 & 1,68 \\
& Cana-de-açúcar & 10,9 & 31,7 & 1,41 \\
\hline
\end{tabular}

Neumann et al. (2004) observaram que a inclusão da silagem de sorgo é equivalente a utilização da silagem de milho, para a produção de novilhos super precoce, além disso, a silagem de sorgo propiciou maior receita liquida por animal confinado, isso ocorreu devido o menor custo de produção.

\section{Conclusões}

As variedades de sorgo apresentam bons índices para a produção animal a pasto, assim como em situações que almejam a utilização de forragem suplementar, no entanto, ainda há necessidade de maiores incentivos para a utilização desse material forrageiro na alimentação animal.

\section{Agradecimentos}

Agradecemos a Universidade Federal do Mato Grosso do Sul pelo apoio ao trabalho e a Coordenação de Aperfeiçoamento de Pessoal de Nível Superior - Brasil (CAPES) - Código de Financiamento 001.

\section{Referências bibliográficas}

Ali, M. A., Abbas, A., Niaz, S., Zulkiffal, M., \& Ali, S. (2009). Morpho-physiological criteria for drought tolerance in sorghum (Sorghum bicolor) at seedling and post-anthesis stages. International Journal of Agriculture and Biology, 11(6), 674-680.

Barbosa, R. A., Nascimento Júnior, D., Euclides, V. P. B., Silva, S. C., Zimmer, A. H., \& Torres Júnior, R. A. (2007). Capim-tanzânia submetido a combinações entre intensidade e frequiência de pastejo. Pesquisa Agropecuária Brasileira, 42(3), 329-340.

Barbosa, R. A., Nascimento Júnior, D., Vilela, H. H., Silva, S. C., Euclides, V. P. B., Sbrissia, A. F., \& Sousa, B. M. L. (2011). Morphogenic and structural characteristics of guinea grass pastures submitted to three frequencies and two defoliation severities. Revista Brasileira de Zootecnia, 40(5), 947-954. DOI: https://doi.org/10.1590/S1516-35982011000500002

Benvenutti, M. A., Gordon, I. J., Poppi, D. P., Crowther, R., \& Spinks, W. (2008). Foraging mechanics and their outcomes for cattle grazing reproductive tropical swards. Applied Animal Behaviour Science, 113(1-3), 15-31. DOI: https://doi.org/10.1016/j.applanim.2007.10.005

Benvenutti, M. A., Gordon, I. J., Poppi, D. P., Crowther, R., Spinks, W., \& Moreno, F. C. (2009). The horizontal barrier effect of stems on the foraging behaviour of cattle grazing five tropical grasses. Livestock Science, 126(1-3), 229-238. DOI: https://doi.org/10.1016/j.livsci.2009.07.006

Bhat, B. V. (2019). Breeding forage sorghum. Breeding Sorghum for Diverse End Uses, 175-191, Hyderabad, India. DOI: https://doi.org/10.1016/B978-0-08-101879-8.00011-5

Chieza, E. D., Arboitte, M. Z., Brondani, I. L., Menezes, L. F. G., Restle, J., \& Santi, M. A. M. (2008). Aspectos agronômicos de híbridos de sorgo (Sorghum bicolor L. Moench) no desempenho e economicidade de novilhos confinados. Acta Scientiarum. Animal Sciences, 30(1), 67-73.

Difante, G. S., Nascimento Júnior, D., Silva, S. C., Euclides, V. P. B., \& Montagner, D. B. (2011). Características morfogênicas e estruturais do capim-marandu submetido a combinações de alturas e intervalos de corte. Revista Brasileira de Zootecnia, 40(5), 955-963. DOI: https://doi.org/10.1590/S1516-35982011000500003

Euclides, V. P. B., Flores, R., Medeiros, R. N., \& Oliveira, M. P. (2007). Diferimento de pastos de braquiária cultivares Basilisk e Marandu, na região do Cerrado. Pesquisa Agropecuária Brasileira, 
42(2), 273-280. DOI: https://doi.org/10.1590/S0100-204X2007000200017

Euclides, V. P. B., Macedo, M. C. M., Valle, C. B. do, Barbosa, R. A., \& Gonçalves, W. V. (2008). Produção de forragem e características da estrutura do dossel de cultivares de Brachiaria brizantha sob pastejo. Pesquisa Agropecuária Brasileira, 43(12), 1805-1812. DOI: https://doi.org/10.1590/S0100-204X2008001200023

Euclides, V. P. B., Macedo, M. C. M., Valle, C. B., Difante, G. S., Barbosa, R. A., \& Cacere, E. R. (2009). Valor nutritivo da forragem e produção animal em pastagens de Brachiaria brizantha. Pesquisa Agropecuária Brasileira, 44(1), 98-106. DOI: https://doi.org/10.1590/S0100204X2009000100014

Fernandes, P. B., Barbosa, R. A., Oliveira, R. T., Oliveira, C. V. V., \& Medeiros-Neto, C. (2020). Defoliation dynamics of Brachiaria brizantha pastures with distinct structural characteristics. Bioscience Journal, 36(1), 203-211. DOI: https://doi.org/10.14393/BJ-v36n1a2020-42211

Fonseca, L., Mezzalira, J. C., Bremm, C., \& Carvalho, P. C. F. (2012). Grazing by horizon: what would be the limits to maintain maximum short-term herbage intake rate. Grassland Science in Europe, 17, 237-239.

Galvão, J. R., Fernandes, A. R., Siva, V. F. A., Pinheiro, D. P., \& Melo, N. C. (2015). Adubação potássica em híbridos de sorgo forrageiro cultivados em sistemas de manejo do solo na Amazônia oriental. Revista Caatinga, 28(4), 70-79. DOI: https://doi.org/10.1590/1983-21252015v28n408rc

Gastal, F., \& Lemaire, G. (2015). Defoliation, shoot plasticity, sward structure and herbage utilization in pasture: Review of the underlying ecophysiological processes. Agriculture, 5(4), 1146-1171. DOI: https://doi.org/10.3390/agriculture5041146

Lemaire, G., Silva, S. C., Agnusdei, M., Wade, M., \& Hodgson, J. (2009). Interactions between leaf lifespan and defoliation frequency in temperate and tropical pastures: a review. Grass and Forage Science, 64(4), 341-353. DOI: https://doi.org/10.1111/j.1365-2494.2009.00707.x

Macêdo, A. J. S., Ramos, J. P. F., Santos, E. M., Sousa, W. H., Oliveira, F. G., Souza, J. T. A., \& Oresca, D. (2018). Morphometric and productive characteristics of sorghum genotypes for forage production in the Brazilian semi-arid. Revista Brasileira de Saúde e Produção Animal, 19(3), 256-267. DOI: https://doi.org/10.1590/s1519-99402018000300003

Mazzanti, A., Lemaire, G., \& Gastal, F. (1994). The effect of nitrogen fertilization upon the herbage production of tall fescue swards continuously grazed with sheep. 1. Herbage growth dynamics. Grass and Forage Science, 49(2), 111-120. DOI: https://doi.org/10.1111/j.1365-2494.1994.tb01983.x

Menezes, M. B., Paiva, L. M., Fernandes, B. P., Campos, N. R. F., Barbosa, R. A., Bento, A. L. L., Rocha, R. F., \& Morais, M. G. (2019). Tissue flow and biomass production of piatã grass in function of defoliation frequency and nitrogen fertilization. Colloquium Agrariae, 15(2), 92-100. DOI: 10.5747/ca.2019.v15.n2.a288ca

Montagner, D. B., Nascimento Júnior, D., Sousa, B. M. L., Vilela, H. H., Euclides, V. P. B., Silva, S. C. da, Sbrissia, A. F., \& Carloto, M. N. (2011). Morphogenetic and structural characteristics of tillers of guinea grass of different age and grazing severities. Revista Brasileira de Zootecnia, 40(10), 21052110. DOI: https://doi.org/10.1590/S1516-35982011001000006

Neumann, M., Restle, J., Alves Filho, D. C., Bernardes, R. A. C., Arboite, M. Z., Cerdótes, L., \& Peixoto, L. A. D. (2002). Avaliação de diferentes híbridos de sorgo (sorghum bicolor, L. Moench) quanto aos componentes da planta e silagens produzidas. Revista Brasileira de Zootecnia, 3l(1 SUPPL.), 302-312. DOI: https://doi.org/10.1590/S1516-35982002000200003

Neumann, M., Restle, J., \& Brondani, I. L. (2004). Avaliação de silagens de sorgo (Sorghum bicolor, L. Moench) ou milho (Zea mays, L.) na produção do novilho superprecoce. Revista Brasileira de Milho e Sorgo, 3(3), 438-452. DOI: https://doi.org/10.18512/1980-6477/rbms.v3n03p\%25p

Oliveira, C. V. V., Barbosa, R. A., Oliveira, R. T., de Almeida, E. M., Paludo, F., Lima, J. A., \& Fernandes, P. B. (2020). The tissue flow in Brachiaria brizantha pasture under intermittent stocking. Journal of Agricultural Studies, 8(1), 9-17. DOI: https://doi.org/10.5296/jas.v8i1.15441

Pacheco, R. F., Alves Filho, D. C., Brondani, I. L., Nornberg, J. L., Pizzuti, L. A. D., \& Callegaro, Á. M. (2014). Características produtivas de pastagens de milheto ou capim sudão submetidas ao pastejo contínuo de vacas para abate. Ciência Animal Brasileira, 15(3), 266-276. DOI: 
https://doi.org/10.1590/1809-6891v15i324387

Pinto, A. P., Abrahão, J. J. S., Marques, J. A., Nascimento, W. G., Perotto, D., \& Lugão, S. M. B. (2010). Desempenho e características de carcaça de tourinhos mestiços terminados em confinamento com dietas à base de cana-de-açúcar em substituição à silagem de sorgo. Revista Brasileira de Zootecnia, 39(1), 198-203. DOI: https://doi.org/10.1590/S1516-35982010000100026

Rezende, R. P., Golin, H. O., Abreu, V. L. da S., Theodoro, G. F., Franco, G. L., Brumatti, R. C., Fernandes, P. B., Bento, A. L. L., \& Rocha, R. F. A. T. (2020). Does intercropping maize with forage sorghum effect biomass yield, silage bromatological quality and economic viability? Research, Society and Development, 9(4), 46942818. DOI: http://dx.doi.org/10.33448/rsd-v9i4.2818

Ribeiro, M. G., Costa, K. A. P., Silva, A. G., Severiano, E. C., Simon, G. A., Cruvinel, W. S., Silva, V. R., \& Silva, J. T. (2015). Grain sorghum intercropping with Brachiaria brizantha cultivars in two sowing systems as a double crop. African Journal of Agricultural Research, 10(39), 3759-3766. DOI: https://doi.org/10.5897/AJAR2015.9705

Ribeiro, M. G., Costa, K. A. P., Souza, W. F., Cruvinel, W. S., Silva, J. T., \& Santos Júnior, D. R. (2017). Silage quality of sorghum and Urochloa brizantha cultivars monocropped or intercropped in different planting systems. Acta Scientiarum. Animal Sciences, 39(3), 243-250. DOI: https://doi.org/10.4025/actascianimsci.v39i3.33455

Rodrigues, L. S., Alves Filho, D. C., Brondani, I. L., Silva, V. S., Paula, P. C., Adams, S. M., Martini, P. M., \& Cardoso, G. S. (2017). Effect of planting pattern of forage sorghum on forage productivity and heifer performance during finishing phase. Semina: Ciências Agrárias, 38(3), 1527-1540. DOI: $10.5433 / 1679-0359.2017 \mathrm{v} 38 \mathrm{n} 3 \mathrm{p} 1527$

Rosa, J. R. P., Silva, J. H. S., Restle, J., Pascoal, L. L., Brondani, I. L., Alves Filho, D. C., \& Freitas, A. K. (2004). Avaliação do comportamento agronômico da planta e valor nutritive da silagem de diferentes hibridos de milho (Zea mays, L.). Revista Brasileira de Zootecnia, 33(2), 302-312. DOI: https://doi.org/10.1590/S1516-35982004000200005

Santos, M. E. R., Fonseca, D. M., Santos, T. G. B., Silva, S. P., Gomes, V. M., \& Silva, G. P. (2011). Morphogenic and structural characteristics of tillers on areas with signalgrass pasture varying on height. Revista Brasileira de Zootecnia, 40(3), 535-542. DOI: http://dx.doi.org/10.1590/S151635982011000300010

Silva, W. L., Basso, F. C., Ruggieri, A. C., Vieira, B. R., Alves, P. L. C. A., \& Rodrigues, J. A. S. (2012). Características morfogênicas e estruturais de híbridos de sorgo submetidos a adubação nitrogenada. Revista Brasileirade Ciencias Agrarias, 7, 691-696. DOI: http://dx.doi.org/10.5039/agraria.v7i4a1698

Simili, F. F., Lima, M. L. P., Moreira, A. L., Soares, P. V., Roma Júnior, L. C., \& Reis, R. A. (2011). Forage mass production and grazing loss of sorghum hybrid in response to the density of the sowing and the spacing between planting lines. Revista Brasileira de Zootecnia, 40(7), 1474-1479. DOI: https://doi.org/10.1590/S1516-35982011000700011

Simili, F. F., Reis, R. A., Furlan, B. N., Paz, C. C. P., Lima, M. L. P., \& Bellingieri, P. A. (2008). Resposta do híbrido de sorgo-sudão à adubação nitrogenada e potássica: composição química e digestibilidade in vitro da matéria orgânica. Ciência e Agrotecnologia, 32, 474-480. DOI: http://dx.doi.org/10.1590/S1413-70542008000200020

Wang, C., Zhou, L., Zhang, G., Xu, Y., Zhang, L., Gao, X., Gao, J., Jiang, N., \& Shao, M. (2017). Optimal fertilization for high yield and good quality of waxy sorghum (Sorghum bicolor L. Moench). Field Crops Research, 203, 1-7. DOI: https://doi.org/10.1016/j.fcr.2016.12.009

Licenciamento: Este artigo é publicado na modalidade Acesso Aberto sob a licença Creative Commons Atribuição 4.0 (CC-BY 4.0), a qual permite uso irrestrito, distribuição, reprodução em qualquer meio, desde que o autor e a fonte sejam devidamente creditados. 